Pareto Optima and Competitive Equilibria with Adverse Selection and Moral Hazard Author(s): Edward C. Prescott and Robert M. Townsend

Source: Econometrica, Vol. 52, No. 1 (Jan., 1984), pp. 21-46

Published by: The Econometric Society

Stable URL: http://www.jstor.org/stable/1911459

Accessed: 09/12/2010 04:08

Your use of the JSTOR archive indicates your acceptance of JSTOR's Terms and Conditions of Use, available at http://www.jstor.org/page/info/about/policies/terms.jsp. JSTOR's Terms and Conditions of Use provides, in part, that unless you have obtained prior permission, you may not download an entire issue of a journal or multiple copies of articles, and you may use content in the JSTOR archive only for your personal, non-commercial use.

Please contact the publisher regarding any further use of this work. Publisher contact information may be obtained at http://www.jstor.org/action/showPublisher?publisherCode=econosoc.

Each copy of any part of a JSTOR transmission must contain the same copyright notice that appears on the screen or printed page of such transmission.

JSTOR is a not-for-profit service that helps scholars, researchers, and students discover, use, and build upon a wide range of content in a trusted digital archive. We use information technology and tools to increase productivity and facilitate new forms of scholarship. For more information about JSTOR, please contact support@jstor.org. 


\title{
PARETO OPTIMA AND COMPETITIVE EQUILIBRIA WITH ADVERSE SELECTION AND MORAL HAZARD ${ }^{1}$
}

\author{
By Edward C. Prescott and Robert M. Townsend
}

\begin{abstract}
This paper explores the extent to which standard, general equilibrium analysis of Pareto optima and of competitive equilibria can be applied to environments with moral hazard and adverse selection problems. Allowing for lotteries, contracts with random components, we first establish that an adverse-selection insurance economy, a moral-hazard insurance economy, a signaling economy, and a private-information labor market economy are all special cases of a simple, general structure. We then show that techniques for characterizing Pareto optimal contracts as solutions to concave programming problems are useful and nice and appear to be broadly applicable; allowing for lotteries, we show how to characterize the optimal allocations for the adverse-selection insurance and labor market economies. We then show that standard existence and optimality theorems for competitive equilibria apply in the linear space containing lotteries if agents with characteristics which are distinct and privately observed at the time of initial trading enter the economy-wide resource constraints in a homogeneous way (other kinds of diversity are not critical). For economies with moral hazard which satisfy the homogeneity condition, competitive contract markets single out a subset of the optima and thus can be consistent with apparent unemployment and with a random allocation of labor supplied though all households are averse to risk. The adverse-selection insurance and signaling economies, however, do not satisfy the homogeneity condition and are difficult to decentralize efficiently with a price system.
\end{abstract}

\section{INTRODUCTION}

THE PURPOSE OF THIS PAPER is to explore the extent to which standard, general equilibrium analysis of Pareto optima and of competitive equilibria can be applied to economies with moral hazard and adverse selection problems. In these economies the information structure is explicit but private. Of particular interest are the Rothschild-Stiglitz [21], Wilson [23] insurance economy, in which each agent observes a parameter indicating the probability of suffering a loss, that is, whether he is a high risk or low risk agent; a Spence [22] signaling economy, in which each agent observes a parameter indicating his inherent productivity as well as the direct or indirect disutility of some unproductive activity; a moral hazard insurance economy in which agents can take an unobserved action determining the probability of suffering a loss; and a private-information labor market economy in which households suffer unobserved shocks to preferences (or to an underlying household production function).

We proceed by showing in Section 2 that despite the apparent diversity among the above-mentioned economies, each can be viewed as a special case of a simple general structure with convex constraints and preferences. This is accomplished

\footnotetext{
'Earlier drafts of this paper have been presented at the NBER Conference on Theoretical Industrial Organization, Montreal, October, 1979, the 4th World Congress Meetings of the Econometric Society, Aix-en-Provence, August, 1980, and the Summer Meetings of the Econometric Society, San Diego, June, 1981. Helpful comments from Ted Groves, Roy Radner, John Roberts, Charles Wilson, our colleagues, the participants of these seminars, and a referee are all gratefully acknowledged. We also thank the National Science Foundation and the Alfred P. Sloan Foundation for financial support.
} 
by considering consumption lotteries indexed by time and by privately-observed shocks and agent types. The constraint set then naturally includes certain incentive compatibility conditions, following the seminal work of Hurwicz [16]; these ensure that it is not in the interest of agents to misrepresent their private information.

Section 3 establishes that Pareto optimal allocations can be determined as solutions to the problem of maximizing weighted averages of the agent-type utilities subject to the incentive-compatibility conditions and resource constraints, a concave programming problem. This result is then used to characterize the optima for both the adverse-selection insurance and the private-information labor market economy. It is established as a by-product that the RothschildStiglitz separating equilibrium is optimal in the space of lotteries under exactly the same conditions which make it optimal in the space of (apparently) deterministic allocations. For the private-information labor market economy it is established, generally, that optima are inefficient in the ex post full-information sense, being consistent with unemployment or overemployment of labor and even with a random allocation of labor though all households are averse to risk. The latter results complement the findings of Green and Kahn [12] and Grossman and Hart [13] on ex post inefficiencies in labor contracts and especially Chari [7] in the use of lotteries.

Section 4 begins an attack on the question of the applicability of classical competitive analysis to economies which can be mapped into our general structure. Previously, Prescott and Townsend [19] established that standard competitive analysis could be applied to a Fisherian dynamic securities economy with private information. For that economy competitive equilibria were shown to exist and to be optimal, in contrast to the problems that have been encountered for other private information economies that also fall within our general structure. With the approach employed by Spence [22] in his studies of signaling economies, for example, there is a multiplicity of equilibria and they generally are not optimal. And with the approach employed by Rothschild-Stiglitz [21], an equilibrium may fail to exist, and again, if one does exist, it may be nonoptimal. We find that the key property upon which standard existence and optimality theorems depend has to do with only one aspect of the information structure of the economy, whether or not agents with characteristics which are distinct and privately observed at the time of initial trading enter the resource constraints in a homogeneous way: preferences, technology, other kinds of ex ante diversity, and ex post diversity are not critical. An implication of this result for economies with signaling opportunities is that nonoptimality, multiplicity, or nonexistence problems do not arise if there is a market for contracts prior to agents knowing their (still privately observed) types. Neither are there problems for the moral hazard insurance economy, nor for the private-information labor market economy.

In Section 4 we illustrate how the private-information labor market economy optimum can be supported in a competitive equilibrium. Each household chooses a contract which maximizes its expected utility subject to its budget constraint. The contract entails options which can be exercised at the discretion of the 
household and involves commitments to supply labor in return for the consumption good. Firms in our labor market economy are viewed as producerintermediaries which make commitments to hire labor and produce the consumption good. Again, the commitments are priced in a competitive market and firms maximize profits. The implication of this section is that unemployment, overemployment, and random assignments, being consistent with optimal contracts, are also consistent with competitive equilibrium allocations. We have, in effect, taken a step toward a synthesis of the implicit labor contract paradigm of Azariadas [4], Bailey [5], and Gordon [11] with standard competitive analysis.

The final section of the paper briefly reports on our efforts to secure standard existence and optimality theorems for all economies consistent with the general structure and offers an instructive contrast to our results for the privateinformation labor market economy (among others). Apparently, there can be a fundamental (unavoidable) adverse selection problem if agents with characteristics which are distinct and privately observed at the time of initial trading enter the economy-wide resource constraints in a heterogenous way, as is the case for the adverse selection insurance and the signaling economies.

\section{THE ECONOMIES}

\section{Basic Mathematical Structure}

There are a finite number of agent types $i=1, \ldots, I$ and a continuum of each type. The fraction of agents of type $i$ is denoted by $\lambda_{i}$. The commodity space is a linear space $L$ and the common consumption possibility set for each agent type, $\bar{X} \subset L$, is closed and convex. The utility function of each agent of type $i$, $u_{i}: \bar{X} \rightarrow R$, is concave (and frequently linear). The endowment of each agent is $\xi \in L$, the same for all agent types. Each agent's type is private information. The commodity space, consumption set, utility functions, and endowment will all be given more precise interpretations in the example-economies which follow.

Let $x_{i} \in L$ be a consumption allocation to each agent of type $i$. Let $r_{i k}$ be a real-valued linear function on $L(k=1,2, \ldots, K ; i=1,2, \ldots, I)$. Then society is subject to resource constraints of the form

$$
\sum_{i} \lambda_{i} r_{i k}\left(x_{i}-\xi\right) \leq 0 \quad(k=1, \ldots, K) .
$$

Note that we allow there to be more than one constraint; the private-information securities economy of Prescott and Townsend [19] provides an example.

An $I$-tuple $x=\left(x_{i}\right)$ of elements belonging to $L$ is implementable if

$$
\begin{aligned}
& x_{i} \in \bar{X}, \quad \text { all } i, \\
& u_{i}\left(x_{i}\right) \geq u_{i}\left(x_{j}\right), \quad \text { all } i, j, \\
& \sum_{i} \lambda_{i} r_{i k}\left(x_{i}-\xi\right) \leq 0, \quad \text { all } k .
\end{aligned}
$$


The first requirement is that the consumption vector belong to the individuals' consumption possibility set. The second is that each individual of type $i$ weakly prefer $x_{i}$ to all the other $x_{j}$. Thus it is not in the interest of any agent to claim to be of some other type. These are the ex ante incentive-compatibility constraints. Certain ex post incentive-compatibility constraints arise naturally in consideration of the set of all allowable contracts (see below) and thus help to determine the common consumption possibility set $\bar{X}$. Justification for restricting attention to the class of allocations satisfying the incentive-compatibility constraints can be found in Harris and Townsend [14, 15] and Myerson [18], building on the seminal work of Hurwicz [16]. The third condition is again the set of resource constraints.

In much of this paper $L$ is assumed to have finite dimension. The assumption that $L$ has finite dimension simplifies the presentation without the loss of anything essential. Then, if both the $u_{i}(\cdot)$ and $r_{i k}(\cdot)$ are linear, we use the dot product to represent them; that is

$$
u_{i} \cdot x_{i} \equiv u_{i}\left(x_{i}\right)=\sum_{l} u_{i l} x_{i l}
$$

and similarly

$$
r_{i k} \cdot x_{i} \equiv r_{i k}\left(x_{i}\right)=\sum_{l} r_{i k l} x_{i l},
$$

where $l$ indexes components of $x_{i} \in L$. (Limiting arguments such as those used in Prescott and Townsend [19] might well be used to establish the results of this paper if $L$ is not finite dimensional.)

We now demonstrate how the well-known adverse-selection insurance, moralhazard insurance, and signaling economies as well as a private-information labor market economy can all be represented in this framework.

\section{Adverse Selection Insurance Economy: $E_{1}$}

Consider the following insurance environment that was considered by Rothschild-Stiglitz [21] and Wilson [23]. There is a continuum of agents; say the set of agents is the unit interval. Each agent of type $i$ receives a random endowment $z$ :

$$
z= \begin{cases}z_{0} & \text { with probability } \theta_{i} \\ z_{1} & \text { with probability }\left(1-\theta_{i}\right)\end{cases}
$$

Here $0<z_{0}<z_{1}$, so when $z=z_{0}$ an agent is said to suffer a loss. This is public information. There are two types of agents by risk class, $i=1,2$, where $0<\theta_{1}$ $<\theta_{2}<1$. Thus the $\theta_{1}$-type agents are the low risk people and $\theta_{2}$-type agents are the high risk people. Each agent's type is private information. Of people of type $i$, $\theta_{i}$ is also the fraction that will suffer a loss. Thus there is no aggregate uncertainty with the fractions of the various types, $\lambda_{i}$, being known. ${ }^{2}$

\footnotetext{
${ }^{2}$ As noted in Prescott and Townsend [19], both here and below, we proceed from an aggregate distribution to individual uncertainty rather than the other way in order to avoid certain well-known mathematics problems associated with measurability. See Bewley and Radner [6].
} 
Each agent has preferences on $C \subset R_{+}$as defined by the utility function $U: R_{+} \rightarrow R$ where $U$ is strictly concave, strictly increasing, and continuously differentiable with $U^{\prime}(0)=\infty$. The points $z_{0}$ and $z_{1}$ belong to $C$. Suppose a consumer is assigned $c_{0}$ if a loss is suffered and $c_{1}$ if one is not. The expected utility for an agent of type $i$ is then

$$
\theta_{i} U\left(c_{0}\right)+\left(1-\theta_{i}\right) U\left(c_{1}\right)
$$

where $c_{0}, c_{1} \in C$.

This environment can be cast in terms of the basic mathematical structure. One approach is to let $L$ be $R^{2}$. Then let $x_{i}$ be a consumption allocation to agents of type $i$ with the first component being $c_{i 0}$ and the second $c_{i 1}$. The common endowment is $\xi=\left(z_{0}, z_{1}\right)$. Then $\bar{X}$ corresponds to $C \times C$ and is closed and convex provided $C$ is closed and convex. The single linear resource constraint is

$$
\sum_{i} \lambda_{i}\left[\theta_{i}\left(c_{i 0}-z_{0}\right)+\left(1-\theta_{i}\right)\left(c_{i 1}-z_{1}\right)\right] \leq 0 .
$$

The incentive compatibility constraints are

$$
\theta_{i} U\left(c_{i 0}\right)+\left(1-\theta_{i}\right) U\left(c_{i 1}\right) \geq \theta_{i} U\left(c_{j 0}\right)+\left(1-\theta_{i}\right) U\left(c_{j 1}\right), \quad \text { all } i, j .
$$

But the space of consumption allocations $\left(x_{i}\right)$ restricted by such constraints is not convex given the strict concavity of $U$.

An alternative approach which results in the utility function being linear, and therefore avoids the nonconvexities associated with the incentive compatibility constraints, is to consider lotteries on $C$. In order that the space $L$ be finite dimensional, the set $C$ is assumed finite with $n$ elements. The lotteries are $n$-dimensional vectors specifying the probability of each point in $C$, say $\mu$ $=(\mu(c))_{c \in C}$ where $\sum_{c} \mu(c)=1$ and $\mu(c) \geq 0$ all $c \in C$. Let $\mu_{0}$ be the lottery if a loss is suffered and $\mu_{1}$ if one is not. The expected utility of $\left(\mu_{0}, \mu_{1}\right)$ for a type $i$ individual is then

$$
W_{i}\left(\mu_{0}, \mu_{1}\right)=\theta_{i} \sum_{c} U(c) \mu_{0}(c)+\left(1-\theta_{i}\right) \sum_{c} U(c) \mu_{1}(c) .
$$

It is also assumed here that fraction $\mu_{0}(c)$ of agents of type $i$ who suffer a loss receive the allocation $c$ and similarly for $\mu_{1}(c)$, so that lotteries introduce no aggregate randomness. The endowment $\xi$ is a pair of probability distributions on $C$, the first one of which assigns probability one to $z_{0} \in C$ and the second probability one to $z_{1} \in C$.

This latter economy can be put into the general mathematical structure as follows. Let $L=R^{2 n}$. Let the first $n$ elements of a consumption vector $x$, denoted $x_{0}$, be the $x_{0 c}=\mu_{0}(c)$ defined above for $c \in C$, and let the second $n$ elements of $x$, denoted $x_{1}$, be the $x_{1 c}=\mu_{1}(c)$ for $c \in C$. The consumption possibility set then requires that $x_{0}$ and $x_{1}$ be probability distributions; that is,

$$
\bar{X}=\left\{x \in L: \sum_{c} x_{0 c}=1, \sum_{c} x_{1 c}=1, x \geq 0\right\} .
$$


Let the first $n$ elements of the utility function $u_{i}$ be the $\theta_{i} U(c)$ for $c \in C$ and the second $n$ elements $\left(1-\theta_{i}\right) U(c)$ for $c \in C$. With these definitions

$$
u_{i}(x)=u_{i} \cdot x=W_{i}\left(\mu_{0}, \mu_{1}\right)
$$

which is just the expected utility to an $i$-type of lottery $\mu_{0}$ if a loss is suffered and lottery $\mu_{1}$ if one is not.

The common endowment $\xi$ is an element of $L$. The resource constraint is that average consumption be less than or equal to the average endowment:

$$
\sum_{i} \lambda_{i}\left[\theta_{i} \sum_{c} x_{i 0 c} c+\left(1-\theta_{i}\right) \sum_{c} x_{i 1 c} c\right] \leq \sum_{i} \lambda_{i}\left[\theta_{i} \sum_{c} \xi_{0 c} c+\left(1-\theta_{i}\right) \sum_{c} \xi_{1 c} c\right] .
$$

As there is a single resource constraint, the $k$ subscript can be dropped on the resource constraint function $r_{i k}$. The first $n$ components of $r_{i}$ are the $\theta_{i} c$ for $c \in C$ and the next $n$ components are $\left(1-\theta_{i}\right) c$. Thus the resource constraint can be put in the form

$$
\sum_{i} \lambda_{i} r_{i} \cdot\left(x_{i}-\xi\right) \leq 0
$$

as required by the general formulation.

\section{Signaling Economy: $E_{2}$}

A particularly interesting class of economic environments are those with signaling opportunities. We consider the following simple signaling economy. The set $C$ is a subset of $R_{+}^{3}$. The first component $c_{1}$ is consumption of goods, the second $c_{2}$ is the signal, and the third $c_{3}$ is consumption of leisure. The utility function for individuals of type $i$ is linear in $c$, and of the form

$$
U_{i}(c)=\theta_{i} c_{1}-c_{2}
$$

where the $\theta_{i}$ have been ordered so that $\theta_{1}<\theta_{2}<\cdots<\theta_{I}$. The fraction of type $i$ is $\lambda_{i}>0$. Again, agent types are private information.

The output of an individual is not observed. A finite fraction, albeit small, of the continuum of individuals is required to produce any output and the resulting productivity of a group is the average of the productivities of the group's members. These assumptions imply it is impossible to deduce anything about an individual's productivity by observing the output of his group. Let $\pi_{i}$ denote the output of the consumption good per unit of labor of individuals of type $i$. Individuals with larger $\theta_{i}$ are more productive so $\pi_{I}>\pi_{I-1}>\cdots>\pi_{1} \geq 0$. The assumption that the signal does not affect output is not crucial and was made for the sake of simplicity. The endowment of leisure time is unity. 
Because agents are risk neutral, we need not consider lotteries; that is, the utility functions are already linear. If they were not risk neutral, following the previous example, it would be necessary to consider lotteries on $C$.

To represent this economy within our basic structure, the commodity space $L$ is $R^{3}$ with component $x_{1}$ as consumption, $x_{2}$ as signal, and $x_{3}$ as leisure consumed. The consumption possibility set is

$$
\bar{X}=\left\{x \in L: x \geq 0, x_{3} \leq 1\right\}
$$

and the utility function for an $i$-type is

$$
u_{i}(x)=\theta_{i} x_{1}-x_{2} .
$$

The endowment $\xi \in L$ is the vector $(0,0,1)$.

The resource constraint is

$$
\sum_{i} \lambda_{i}\left[x_{i 1}+\pi_{i}\left(x_{i 3}-\xi_{3}\right)\right] \leq 0 .
$$

This states that the average consumption $\sum_{i} \lambda_{i} x_{i 1}$ must be less than average production $\sum_{i} \lambda_{i} \pi_{i}\left(\xi_{3}-x_{i 3}\right)$. As for the previous example, there is a single resource constraint, characterized by the vectors $r_{i} \in R^{3}$ where $r_{i}=\left(1,0, \pi_{i}\right)$. With this definition the resource constraint can be put in the form

$$
\sum_{i} \lambda_{i} r_{i} \cdot\left(x_{i}-\xi\right) \leq 0
$$

as required by the general formulation.

\section{A Moral-Hazard Insurance Economy: $E_{3}$}

The adverse-selection insurance economy $E_{1}$ is modified as follows. There is only one type of agent, so we can ignore the $\lambda_{i}$ in what follows, but the probability of loss depends upon a costly, private action of the agent. More precisely, each agent receives a random endowment $z \in Z=\left\{z_{0}, z_{1}\right\}$ with $0<z_{0}$ $\left\langle z_{1}\right.$ and the probability of $z$ given the agent's action $a \in A=\left\{a_{1}, a_{2}, \ldots, a_{m}\right\}$ is $\theta_{z \mid a}$. The realization of $z$ is public, the action taken is not. Also, the larger is action $a$, the smaller is the probability of $\operatorname{loss} \theta_{z_{0} \mid a}$. The interpretation here is that a larger action corresponds to an agent being more careful. This is a standard set-up.

Each agent has preferences on the finite set $C \times A$, where $C$ is a finite subset of $R_{+}$and has $n$ elements. Preferences are defined by a utility function $U(c, a)$ where $U$ is increasing in $c$, decreasing in $a$, and concave. For $(c, a) \in C \times A$, let $U_{c a}=U(c, a)$.

In terms of our basic mathematical structure, let the linear space $L$ be the Euclidean space of dimension $n 2 \mathrm{~m}$. A consumption vector $x$ is a triply indexed element $\left(x_{c z a}\right)$ for $c \in C, z \in Z$, and $a \in A$. The interpretation is as follows. A 
lottery with probabilities $x_{a}$ first determines an action $a$ for each agent. Number $x_{a}$ is also the fraction of agents in the population who are to take action $a$. Conditional on this action $a$, a second lottery with probabilities $x_{c z \mid a}$ determines consumption $c$ and endowment $z$ of the agent. Of course, nature plays a role in this second lottery since the conditional probabilities $\theta_{z \mid a}$ are technologically determined constraints. In fact it is required that

$$
\sum_{c} x_{c z \mid a}=\theta_{z \mid a}
$$

for consistency. Finally, the marginal and conditional distributions $x_{a}$ and $x_{c z \mid a}$ determine the joint distribution $x_{c z a}$ specified above.

Agents have preferences on $\bar{X}$ where

$$
\begin{gathered}
\bar{X}=\left\{x \in L_{+}: \sum_{c, z, a} x_{c z a}=1 ; \theta_{z \mid a} \sum_{c, z} x_{c z a}=\sum_{c} x_{c z a} \text { all } a, z ;\right. \\
\left.\sum_{c, z} U_{c a} x_{c z a} \geq \sum_{c, z} U_{c a^{\prime}} x_{c z a} \frac{\theta_{z \mid a^{\prime}}}{\theta_{z \mid a}} \text { all } a, a^{\prime}\right\} .
\end{gathered}
$$

The first constraint is that the probabilities sum to one. The second is that the probability distribution of $z$ given $a$ (if defined for that probability distribution) equals the technologically determined probability $\theta_{z \mid a}$. The third constraint is to ensure incentive compatibility. This is not obvious and is derived as follows. The commodity point $x$ must be structured such that if $a$ occurs, it is not in the interest of the agent to choose some other action $a^{\prime}$; that is,

$$
x_{a} \sum_{c, z} U_{c a} x_{c z \mid a} \geq x_{a} \sum_{c, z} U_{c a^{\prime}} \operatorname{Pr}\left\{c, z \mid a^{\prime}\right\} .
$$

Here $\operatorname{Pr}\left\{c, z \mid a^{\prime}\right\}$ is the probability of the pair $(c, z)$ given that the agent is subject to lottery $x_{c z a}$ but chooses action $a^{\prime}$. Thus, under $x_{c z a}$

$$
\operatorname{Pr}\left\{c, z \mid a^{\prime}\right\}=x_{c \mid z a} \theta_{z \mid a^{\prime}}=\frac{x_{c z \mid a}}{\theta_{z \mid a}} \theta_{z \mid a^{\prime}} .
$$

By substitution, the above expression holds if and only if

$$
\sum_{c, z} U_{c a} x_{c z \mid a} x_{a} \geq \sum_{c, z} U_{c a^{\prime}} x_{c z \mid a} x_{a} \frac{\theta_{z \mid a^{\prime}}}{\theta_{z \mid a}}
$$

As $x_{c z a}=x_{c z \mid a} x_{a}$, the third constraint indeed ensures incentive compatibility. This particular representation makes clear that set $\bar{X}$ is convex.

Of course, there is also a resource constraint, that average consumption be no greater than average endowment, or

$$
\sum_{c, z, a} x_{c z a}(c-z) \leq 0
$$


This constraint corresponds to constraint (2.3) in the basic structure, with endowment $\xi$ being the zero vector in $L$. Here the single resource constraint takes on a special form,

$$
r \cdot(x-\xi) \leq 0
$$

with $r_{c z a}=c-z$. The vector $r$ is not indexed by $i$.

\section{Private-Information Labor Market Economy: $E_{4}$}

Imagine an economy in which households have preferences over some market produced consumption good $c$ and over labor supply $l$ as represented by a utility function $U_{\theta}(c, l)$ which is continuous, concave, increasing in $c$, and decreasing in $l$. Here $c$ and $l$ are nonnegative real numbers, with a maximal labor supply of $i$. Here also $\theta \in \Theta=\{1,2\}$ is interpreted as one of two possible shocks to the household's preferences at the time of consumption and labor supply. ${ }^{3}$ Each household's shock is private information to the household in the consumption period. It is known that fraction $\lambda_{\theta}$ of households in the population will suffer shock $\theta$ in the consumption period. Thus, from the standpoint of a prior planning period, each household regards $\lambda_{\theta}$ as the probability of suffering shock $\theta$ in the consumption period.

There are a finite number of firms in the economy, each with a technology for transforming labor $l$ into output $q$ of the consumption good in a linear way, namely $q=a l, a>0$; think of a fixed number of industrial projects, or a fixed number of plots of land in an agrarian economy. Firms are owned by households with predetermined profit shares in the population. With the constant returns to scale assumption, however, profits are necessarily zero in a competitive equilibrium and thus will be disregarded in what follows. By the same token, we may act as if there were only one firm.

The natural commodity bundle for households in this model is a consumption, labor-supply pair $(c, l)$. The space of such bundles would be used if trade were restricted to take place subsequent to the realization of household shocks $\theta$. But trade can take place in the prior planning period. So, following Arrow [2] and Debreu [10], it is natural to index the $(c, l)$ bundle by the household-specific shocks. Shocks are private to the household, however, so to circumvent incentive problems, we suppose each individual household is assigned a contract which specifies consumption, labor supply pairs $(c, l)$ under a variety of individually-

\footnotetext{
${ }^{3}$ The introduction of shocks to preferences may seem somewhat artificial. But the economy is readily given a deeper, more satisfactory interpretation. Suppose in particular that labor can be supplied also to a household production function, that is, there is a technology for transforming labor input into output of an idiosyncratic, home-produced good, a good which cannot be transferred among households. Suppose also that the household production function is subject to privatelyobserved technology shocks, $\theta$. Finally, suppose households have preferences over consumption of the market-produced good, the home-produced good, and total labor supply. Then this specification delivers an indirect utility function (as in the text) over consumption of the market-produced good and over market labor supply.
} 
effected contingencies or options. For example, the contract might allow "sick" leaves, voluntary overtime, and so on, in addition to the "work-as-usual" option. (There is no monitoring of underlying circumstances.) The point is that the household itself chooses which option to effect subsequent to its underlying circumstance, its shock $\theta$. Now under any contract an individual household will act in its own best interest, inducing a natural ordering on outcomes, $(c, l)$ pairs, relative to its $\theta$-contingent utility function. Thus, we may adopt a more abstract, canonical representation for any contract, following Harris and Townsend [14, 15], and Myerson [18], and suppose, without loss of generality, that households make direct announcements of their shocks $\theta$ and that contracts are such that these announcements are made truthfully. Finally, we suppose the further possibility that a contract specifies a random $(c, l)$ bundle, conditional on an announcement $\theta$. For example, laborers may report to work under some circumstances, but there need be no guarantee of employment.

More formally then the labor market economy with planning period contracts is cast in terms of the basic mathematical structure as follows. First, to ensure that the commodity space is finite-dimensional, restrict attention to a finite number $n$ of consumption-labor supply pairs $(c, l)$. Then let the commodity space $L$ be $R^{2 n}$. The first $n$ components of a consumption vector $x \in L$, with typical elements, $x_{1}(c, l)$, assign probability to consumption-labor supply pairs conditional upon the announcement $\theta=1$, with a similar interpretation for the second $n$ components and $\theta=2$. Households are alike ex ante in the planning period (but not ex post), so the $\lambda_{i}$ and $i$ may be dropped from the notation. The expected utility of the representative household is then

$$
u(x)=\sum_{\theta} \lambda_{\theta} \sum_{(c, l)} x_{\theta}(c, l) U_{\theta}(c, l) .
$$

Thus, the consumption possibilities set is

$$
\begin{aligned}
& \bar{X}=\left\{x \in L: x \geq 0, \sum_{(c, l)} x_{\theta}(c, l)=1 \text { for } \theta=1,2\right. \text { and } \\
&\left.\sum_{(c, l)} x_{\theta}(c, l) U_{\theta}(c, l) \geq \sum_{(c, l)} x_{\phi}(c, l) U_{\theta}(c, l) \text { for } \theta, \phi \in \Theta\right\} .
\end{aligned}
$$

This ensures both that the $x_{\theta}$ are probability measures for each $\theta$ and that in the consumption period each household will truthfully reveal its shock $\theta$.

The endowment $\xi \in L$ is the element for which $\xi_{\theta}(c, l)=0$ unless $(c, l)=(0,0)$, i.e., $\xi_{\theta}$ puts all probability on the zero point in the underlying commodity space. The resource constraint is that average consumption not exceed average production, that is

$$
\sum_{\theta} \lambda_{\theta} \sum_{(c, l)} x_{\theta}(c, l) c \leq \sum_{\theta} \lambda_{\theta} \sum_{(c, l)} x_{\theta}(c, l) a l .
$$

Here $x_{\theta}(c, l)$ is interpreted as the fraction of agents of type $\theta$ who are assigned the consumption-labor supply pair $(c, l)$. Thus the resource constraint has the 
form

$$
r \cdot(x-\xi) \leq 0
$$

where component $r_{\theta}(c, l)=\lambda_{\theta}(c-a l)$.

\section{PARETO OPTIMA}

Pareto optimal allocations for the general structure can be obtained by maximizing weighted averages of the agent types' utilities. Let the set of possible weights be

$$
\Gamma=\left\{\gamma \in R^{I}: \gamma_{i} \geq 0 \text { and } \sum_{i} \gamma_{i}=1\right\} .
$$

For $\gamma \in \Gamma$, let $\phi(\gamma)$ denote the set of consumption allocations which are solutions to the program

$$
\operatorname{Max}_{x=\left(x_{i}\right)} \sum_{i} \gamma_{i} u_{i} \cdot x_{i}
$$

subject to $x_{i} \in \bar{X}$ all $i, u_{i} \cdot x_{i} \geq u_{i} \cdot x_{j}$ all $i$ and $j$, and $\sum_{i} \lambda_{i} r_{i k} \cdot\left(x_{i}-\xi\right) \leq 0$ all $k$. Thus $\phi(\gamma)$ is a subset of the $I$-cross product space of $L$. Finally, let

$$
\Phi=\bigcup_{\gamma \in \Gamma} \phi(\gamma)
$$

Lemma 3.1: The set $\Phi$ contains all the Pareto optima. If $\gamma>0$ (i.e., $\gamma_{i}>0$ all $i$ ), then all allocations belonging to $\phi(\gamma)$ are optima. Finally, if an I-tuple $x=\left(x_{i}\right)$ belongs to $\phi(\gamma)$ and if $x$ is not Pareto dominated by another element belonging to $\phi(\gamma)$, then $x$ is an optimum.

Proof: The constraints are convex and the objective function linear. Consequently, the utility possibility set is convex. Let $\gamma$ define a supporting hyperplane at the point on the utility possibilities frontier associated with Pareto optimal allocation $x^{*}$. Such a hyperplane exists by the separation theorem. For this $\gamma^{*} \in \Gamma, x^{*}$ is a solution to the program. This proves the first statement of the lemma.

To prove the second statement consider some $\gamma>0$ and some allocation $x \in \phi(\gamma)$. Suppose $x$ can be Pareto dominated. Then $x$ cannot be a solution to the $\gamma$-program. This contradiction establishes that solutions to such programs are Pareto optima.

To prove the last statement of the lemma, let $x$ be a solution to some $\gamma$-program with the specified nondominance property, but suppose $x$ is not an optimum. Then there exists an allocation $x^{\prime}$ which Pareto dominates $x$. By assumption, $x^{\prime}$ does not belong to $\phi(\gamma)$. But the value of $x^{\prime}$ for the $\gamma$-program is at least as great as the value for $x$, and thus $x^{\prime}$ must be a solution to the $\gamma$-program, the desired contradiction. 
TheOREM 3.1: If the set $\bar{X}$ is compact and contains $\xi$, the set of Pareto optimal allocations is nonempty.

Proof: A feasible solution exists namely $x_{i}=\xi$ for all $i$ so the constraint set is nonempty. The objective function is linear and therefore continuous. As the resource and incentive constraints are closed, a continuous function is being maximized on a compact set. Consequently for any $\gamma \in \Gamma$, a solution to the program exists. By the lemma, a solution to a $\gamma$-program is necessarily an optimum if $\gamma>0$.

Our examples with lotteries all assume that the underlying consumption possibilities set $C$ has a finite number of points. This is sufficient to ensure that set $\bar{X}$ is compact, a result that is used in the existence argument. There is a straightforward extension of the result to the case of $C$ being a compact separable metric space, for example, a closed and bounded subset of $R^{n}$ with the Euclidean metric; with the weak topology, the set of probability measures on the Borel subsets of $C$ is compact (under these weaker conditions) and the functions defining the objective and constraints are continuous. When the constraint set is defined by the finite set of linear inequalities (other than the nonnegativity constraints), a stronger version of Lemma 3.1 holds; then every Pareto optimal allocation is the solution to a program with all components of $\gamma$ positive. ${ }^{4}$

\section{Pareto Optima for the Adverse-Selection Insurance Economy}

Rothschild and Stiglitz [21] demonstrate, under certain conditions, that their separating equilibrium both exists and is an optimum within a more limited class of allocations than the one we consider. One question that will be answered is whether that allocation is an optimum within our larger class of allocations. The principal result, however, is the complete specification of the set of Pareto optima for this insurance economy.

Let $\bar{z}$ be the ex post per capita endowment, so

$$
\bar{z}=\sum_{i} \lambda_{i}\left[\theta_{i} z_{0}+\left(1-\theta_{i}\right) z_{1}\right]
$$

Per capita consumption is constrained by this quantity. With this definition, the program for determining the Pareto optima for $\gamma \in \Gamma$ is the linear program

$$
\begin{aligned}
& \operatorname{Max} \sum_{i} \gamma_{i} \sum_{c} U(c)\left[x_{i 0 c} \theta_{i}+x_{i 1 c}\left(1-\theta_{i}\right)\right], \\
& x_{1}, x_{2} \geq 0,
\end{aligned}
$$

where $x_{i 0 c}$ is the probability of a type $i$ consuming $c \in C$ conditional on a loss and $x_{i 1 c}$ is the probability of a type $i$ consuming $c$ conditional on no loss, $i=1,2$.

\footnotetext{
${ }^{4}$ We would like to thank an anonymous referee for pointing out this result.
} 
The constraints are

$$
\sum_{c} U(c)\left[\left(x_{10 c}-x_{20 c}\right) \theta_{2}+\left(x_{11 c}-x_{21 c}\right)\left(1-\theta_{2}\right)\right] \leq 0
$$

(agents of type two weakly prefer $x_{2}$ to $x_{1}$ );

$$
\sum_{c} U(c)\left[\left(x_{20 c}-x_{10 c}\right) \theta_{1}+\left(x_{21 c}-x_{11 c}\right)\left(1-\theta_{1}\right)\right] \leq 0
$$

(agents of type one weakly prefer $x_{1}$ to $x_{2}$ );

$$
\sum_{i, c} \lambda_{i} c\left[x_{i 0 c} \theta_{i}+x_{i 1 c}\left(1-\theta_{i}\right)\right] \leq \bar{z}
$$

(this is the single resource constraint);

$$
\sum_{c} x_{10 c}=1
$$

$$
\sum_{c} x_{11 c}=1
$$

$$
\sum_{c} x_{20 c}=1
$$

$$
\sum_{c} x_{21 c}=1 \quad \text { (probabilities sum to one). }
$$

Letting $\mu_{k}$ denote the Lagrange multiplier associated with constraint (3.k), differentiating with respect to the $x_{10 c}$ yields the first-order conditions,

$$
\gamma_{1} U(c) \theta_{1}-\mu_{1} U(c) \theta_{2}+\mu_{2} U(c) \theta_{1}-\mu_{3} c \theta_{1} \lambda_{1}+\mu_{4} \leq 0
$$

for all $c \in C$. Analogous first-order conditions hold for the $x_{20 c}$, the $x_{11 c}$ and the $x_{21 c}$.

Constraint (3.8) must hold with equality for some $c \in C$. Otherwise all the $x_{10 c}$ would be zero and that would violate constraint (3.4). The left-hand side of (3.8) can be viewed as a function of $c$. Thus, the Lagrange multipliers must be such that this function has a maximum (of zero) at points at which condition (3.8) holds as an equality. Now suppose the set $C$ has an arbitrarily large number of elements, so that the maximal distance between any point and its nearest neighbor is arbitrarily small. Also recall that $u(\cdot)$ is strictly concave. Then if

$$
\gamma_{1} \theta_{1}-\mu_{1} \theta_{2}+\mu_{2} \theta_{1} \leq 0
$$

the left-hand side of (3.8) is a strictly decreasing convex function of $c$ and so attains a maximum at $c=0$. If

$$
\gamma_{1} \theta_{1}-\mu_{1} \theta_{2}+\mu_{2} \theta_{1}>0
$$

the left-hand side of (3.8) is strictly concave function of $c$ and so attains a maximum at a single point (on the assumption that the set $C$ can be made 
arbitrarily large). In summary the result is that $x_{10 c}$ equals one for some $c \in C$ and zero otherwise. By precisely the same argument, probability measures $x_{11}$, $x_{20}$, and $x_{21}$ place all their mass on single points denoted by $c_{11}, c_{20}$, and $c_{21}$, respectively. These points depend upon the weights $\gamma$ chosen.

One implementable allocation is for everyone to consume $\bar{z}$ independent of their realized endowment. The utility for this allocation is $U(\bar{z})$ for everyone. If one agent type realizes expected utility exceeding $U(\bar{z})$, that type's expected consumption, by Jensen's inequality, must exceed $\bar{z}$. This implies via the resource constraint that the expected consumption of the other type agents is less than $\bar{z}$ and, by Jensen's inequality, their expected utility less than $U(\bar{z})$.

We divide the Pareto optima into three sets. The first is for everyone to consume $\bar{z}$ with certainty. The second set contains those optima for which the expected utility of type one agents exceeds $U(\bar{z})$ and the third are those for which the expected utility of type one agents is less than $U(\bar{z})$.

It can be established that for set two there is no uncertainty in consumption for type two agents (i.e., $c_{20}=c_{21}=c_{2}$ ). Suppose the contrary. By eliminating uncertainty in the consumption of type two agents (if there is any) while preserving their expected consumption, the utility of type two agents is increased, the resource constraint continues to be satisfied, and slack is introduced into constraint (3.1). Note that constraint (3.2) continues to be satisfied: expected consumption of type two agents is less than $\bar{z}$ in set two so the type one agents strictly prefer their allocation $x_{1}$ which yields expected utility greater than $U(\bar{z})$ to the no uncertainty $x_{2}$ allocation. This also establishes that $\mu_{2}=0$ in set two. And $c_{10} \neq c_{11}$ as well, for otherwise (3.1) would be violated.

Actually constraint (3.1) is binding in set two for otherwise $c_{10}$ and $c_{11}$ could be made more nearly equal while preserving the expected consumption of type one. This increases expected utility of type one. Resource constraint (3.3) is also binding for if it were not, by increasing $c_{2}$ a little, expected utility of type two agents could be increased without violating any constraint. Since (3.1) and (3.3) are binding, $c_{10}$ and $c_{11}$ must satisfy

$$
\theta_{2} U\left(c_{10}\right)+\left(1-\theta_{2}\right) U\left(c_{11}\right)=U\left(c_{2}\right)
$$

and

$$
\lambda_{1}\left[\theta_{1} c_{10}+\left(1-\theta_{1}\right) c_{11}\right]+\lambda_{2} c_{2}=\bar{z}
$$

for elements of Pareto optima set two. To find the solution to (3.10) and (3.11), consider the space of $\left(c_{10}, c_{11}\right)$ pairs. The point $c_{2}$ lies in this space on the $45^{\circ}$ line below the negatively sloped line (3.11). (Recall $c_{2}<\bar{z}$.) Thus the negatively sloped line (3.11) intersects the indifference curve for which (3.10) is satisfied at two points. The better of the solutions for agents of type one is the one for which $c_{10}<c_{2}<c_{11}$. Subsequently, $c_{10}$ and $c_{11}$ are functions of $c_{2}$ as the better solution to (3.10) and (3.11).

This nearly completes the specification of the allocations in Pareto optima set two. There, however, is the condition that the Lagrange multipliers $\mu_{1}$ and $\mu_{3}$ be 
positive. We exploit this condition with the additional assumption that all the optimal allocations are interior points of the set $C$ and that the set $C$ is sufficiently large to insure that such allocations satisfy the appropriate conditions for maxima as if $C$ were a continuum. Thus, given that $\mu_{2}=0$ and that $c_{20}=c_{21}=c_{2}$, from (3.8) and the analogous first-order conditions

$$
\begin{aligned}
& {\left[\gamma_{1}-\mu_{1} \theta_{2} / \theta_{1}\right] U^{\prime}\left(c_{10}\right)=\mu_{3} \lambda_{1},} \\
& {\left[\gamma_{1}-\mu_{1}\left(1-\theta_{2}\right) /\left(1-\theta_{1}\right)\right] U^{\prime}\left(c_{11}\right)=\mu_{3} \lambda_{1},} \\
& {\left[\gamma_{2}+\mu_{1}\right] U^{\prime}\left(c_{2}\right)=\mu_{3} \lambda_{2}=\mu_{3}\left(1-\lambda_{1}\right),} \\
& \gamma_{1}+\gamma_{2}=1 .
\end{aligned}
$$

Given $c_{2}$, these are four linear equations in the unknowns $\mu_{1}, \mu_{3}, \gamma_{1}$, and $\gamma_{2}$. (Remember $c_{10}$ and $c_{11}$ are functions of $c_{2}$ being the better solution to (3.10) and (3.11).) An additional requirement is that the solution to (3.12) (which exists) be nonnegative. It is tedious to establish, but this requirement is that

$$
\frac{\lambda_{2}}{\lambda_{1}} \frac{\theta_{2}-\theta_{1}}{\theta_{1}\left(1-\theta_{1}\right)} \geq \frac{U^{\prime}\left(c_{2}\right)\left[U^{\prime}\left(c_{10}\right)-U^{\prime}\left(c_{11}\right)\right]}{U^{\prime}\left(c_{10}\right) U^{\prime}\left(c_{11}\right)} .
$$

As $c_{2}$ approaches $\bar{z}$, the distance between $c_{10}$ and $c_{11}$ goes to zero. Therefore, given that $U$ is continuously differentiable, for $c_{2}$ sufficiently near $\bar{z}$, this inequality is satisfied. Thus, Pareto optima set two is nonempty. Finally, let $\bar{c}_{2}$ be the minimal level for which inequality (3.13) is satisfied. Then it holds for all $\bar{c}_{2} \leq c_{2}<\bar{z}$. If this were not the case, the utility possibility set would not be convex.

The argument for characterizing optima set three is symmetric with respect to the agents' types with some obvious exceptions necessitated by the fact that $\theta_{2}>\theta_{1}$. To characterize optima for set three interchange subscripts for the two agent types with the exception that one uses the solution to (3.10) and (3.11) for which $c_{20}>c_{21}$ and the direction of inequality (3.13) is reversed.

As condition (3.10), (3.11), and (3.13) along with the additional requirement that the contracts be actuarially fair are just those for the optimality of the Rothschild-Stiglitz separating equilibrium allocation, that allocation is Pareto optimal in our larger class of allocations as well.

Randomness in consumption is used to separate the agents. The agent type realizing the higher expected utility incurs the uncertainty. The cost of this uncertainty is less to agents of that type and they are more than compensated for it by higher expected consumption.

\section{Pareto Optima for the Private-Information Labor Market Economy}

This subsection makes the point that the introduction of private information alone into an otherwise standard environment can produce not only apparent inefficiencies such as unemployment of labor but also a random assignment of 
labor supplied in a world where all households are averse to risk. The key to this conclusion is that ex post full-information inefficiencies and randomness are both consistent with ex ante private-information optimal allocations.

The program for determining the optima for the private-information labor market economy is the linear program

$$
\operatorname{Max}_{x_{\theta}(c, l)} \sum_{\theta} \lambda_{\theta} x_{\theta}(c, l) U_{\theta}(c, l)
$$

subject to

$$
\begin{aligned}
& \sum_{(c, l)} x_{1}(c, l) U_{1}(c, l) \geq \sum_{(c, l)} x_{2}(c, l) U_{1}(c, l), \\
& \sum_{(c, l)} x_{2}(c, l) U_{2}(c, l) \geq \sum_{(c, l)} x_{1}(c, l) U_{2}(c, l), \\
& \sum_{\theta} \lambda_{\theta} \sum_{(c, l)} x_{\theta}(c, l) a l \geq \sum_{\theta} \lambda_{\theta} \sum_{(c, l)} x_{\theta}(c, l) c, \\
& \sum_{(c, l)} x_{1}(c, l)=1 \\
& \sum_{(c, l)} x_{2}(c, l)=1 .
\end{aligned}
$$

Here (3.21) and (3.22) are the relevant incentive compatibility constraints, those which will cause household of type $\theta$ to truthfully announce it is of type $\theta$. Constraint (3.23) is the resource constraint while (3.24) and (3.25) require that the probabilities sum to one.

In this subsection, attention is restricted to economies in which the utility functions are separable having the form

$$
U_{\theta}(c, l)=V(c)-W_{\theta}(l) .
$$

The function $V(\cdot)$ is strictly increasing and strictly concave with $V^{\prime}(0)=\infty$. The functions $W_{\theta}$ are strictly increasing and convex. Agents of type two are assumed uniformly more risk averse with respect to labor supply; that is, $W_{1}^{\prime \prime}(l)<W_{2}^{\prime \prime}(l)$ for all $0 \leq l \leq \bar{l}$. It is also supposed for simplicity that $\lambda_{1}=\lambda_{2}=1 / 2$.

An upper bound for the value of the program is obtained when the program without the incentive constraints is solved. This latter program is easily solved for an optimum exists in the space of deterministic allocations (degenerate lotteries). An interior solution for this less constrained program satisfies

$$
\begin{aligned}
& V^{\prime}\left(c_{1}\right)=V^{\prime}\left(c_{2}\right), \\
& W_{\theta}^{\prime}\left(l_{\theta}\right)=a V^{\prime}\left(c_{\theta}\right) \quad \text { for } \quad \theta=1,2 .
\end{aligned}
$$


Here $c_{\theta}$ and $l_{\theta}$ are the deterministic consumption and labor supply for type $\theta$. Thus at an interior solution to the less constrained program the marginal utility of consumption and the marginal rate of substitution of consumption for labor supply are equal across agent types, with the latter equal to the marginal product of labor, $a$. Of course, the resource constraint will be satisfied at equality: $c_{1}+c_{2}=a l_{1}+a l_{2}$.

An interior solution to the unconstrained program generally will not satisfy the incentive constraints. To see this, note that in such a solution consumptions are necessarily equal across agent types, say, equal to $c$. So, to satisfy the incentive constraints labor supplied must be equal as well, say, equal to $l$. Then from the resource constraint, $c=a l$. But the points at which $W_{\theta}^{\prime}(l)=a V^{\prime}(a l)$ generally will differ for the two $\theta$ types. Thus, with incentive constraints imposed, the best deterministic allocation yields value strictly less than the optima when they are not imposed. We now introduce lotteries into labor supply and make the point that with differences in risk aversion such lotteries can lessen the impact of incentive constraints.

This is well illustrated by the following parametric specification. Suppose $V(c)=\ln (c), W_{1}(l)=l$, and $W_{2}(l)=l^{2} / 3$. Thus, households of type one ex post are risk neutral in labor supply. Without the incentive constraints, the solution to the program would be $c_{1}=c_{2}=a, l_{1}=1 / 2$, and $l_{2}=3 / 2$. This allocation violates the incentive constraints, for type two would claim to be of type one. But, randomness can be introduced into the labor supply of type one. This does not affect the utility of type ones as they are risk neutral in labor supply. This randomness does make the allocation to type one less attractive to type two. Consider the following allocations. With certainty set $c_{1}=c_{2}=a$ and $l_{2}=3 / 2$. Set $l_{1}=0$ with probability $(1-\alpha)$ and set $l_{1}=\dot{l}$ with probability $\alpha$ where $\alpha$ satisfies $(1-\alpha) \cdot 0+\alpha \vec{l}=1 / 2$. This allocation satisfies all the constraints and yields the same value as the program absent the incentive constraints for sufficiently large $\bar{l}$.

The example just described illustrates in a dramatic way the gain to lotteries; here the utility of a full-information optimum can be achieved, though, in general, we think full-information optimality an inappropriate welfare criterion. But the example is unsatisfactory for this subsection in that labor supply is not $e x$ post inefficient; despite the randomness, the marginal rate of substitution of consumption for labor supply for all households equals the marginal product of labor.

We now establish that there must be randomness in labor supply for economies sufficiently close to this special economy with all types strictly risk averse in labor supply. We also establish that there can be no randomness in consumption for either type. With randomness in a type's labor supply, otherwise identical agents supply different quantities of labor. Thus, with constant consumption, the ex post marginal rate of substitution between consumption and labor supply cannot equal the corresponding marginal rate of transformation for all agents of that type. 
We first establish that for an optimum the lottery on consumption is degenerate. Among the first-order conditions for the above-given program are

$$
\begin{aligned}
& \left(\lambda_{1}+\mu_{1}-\mu_{2}\right) V(c)-\mu_{3} \lambda_{1} c-\left(\lambda_{1}+\mu_{1}\right) W_{1}(l)+\mu_{2} W_{2}(l)+\mu_{3} \lambda_{1} a l+\mu_{4} \\
& \quad \leq 0
\end{aligned}
$$

for all $(c, l)$ where the Lagrange multipliers are numbered in accord with equation $(3.2 j)$ of the constraints. This condition must hold with equality for some $(c, l)$ pair. To discover where equality is achieved, it is convenient to view the left-hand side of (3.26) as a function of $c$ and $l$. If $\left(\lambda_{1}+\mu_{1}-\mu_{2}\right) \leq 0$, the left-hand side of (3.26) would be a convex, strictly decreasing function of $c$, and so would achieve a maximum with respect to $c$, holding $l$ fixed, at $c=0$. Thus, consumption would be deterministic at $c=0$, but this cannot be a solution with $V^{\prime}(0)=\infty$. Thus $\left(\lambda_{1}+\mu_{1}-\mu_{2}\right)>0$ and the left-hand side of (3.26) achieves a unique maximum with respect to $c$ at some $c_{1}>0$ independent of $l$ (on the assumption that the distance between adjacent $c$ 's can be made arbitrarily small). By a similar argument, consumption of type two households is deterministic at some $c_{2}>0$.

We now establish that for some economies there must be randomness in labor supply for some agent type. We assume $V(c)=\ln (c), W_{2}(l)=l^{2} / 3$, and $W_{1}(l)$ $=l^{\beta}, 1 \leq \beta$. The limit as $\beta$ approaches one is the parametric example considered previously. For the limit $\beta=1$, we have shown that the best deterministic allocation yields an expected utility that is strictly less than the value of the optimum in the space of lotteries. It can be established by a careful continuity argument that this must also be true for all $\beta$ in some open right-neighborhood of one. This establishes the need for lotteries on labor supply for some economies with all types strictly risk averse.

The randomness in labor supply requires that at the deterministic optimum, the agent supplying less labor be sufficiently less risk averse in labor supply. Then, by introducing randomness in that type's labor supply and reducing differences in consumption between types, the incentive problems can be lessened and expected ex ante utility increased.

\section{COMPETITIVE CONTRACT MARKETS IN ECONOMIES WITH MORAL-HAZARD}

In this section we define a standard competitive equilibrium in our commodity space. That is, we define the price system on the linear space $L$, and optimal actions are defined relative to that price system. In equilibrium, consumption choices are maximal in the budget set, a production choice maximizes profits in the production possibilities set, and markets clear. Thus the economy is decentralized in the usual way. As it turns out, such a competitive equilibrium construct is successful in the moral hazard insurance economy, the privateinformation labor market economy, and virtually any other economy in which the coefficients $r_{i k}$ of the resource constraint (2.3) do not depend on $i$, that is, 
$r_{i k}=r_{k}$ for all $i$. That is, in such environments competitive equilibria exist, are optimal, have a natural market interpretation, and yet are capable of explaining apparent nonmarket clearing phenomena. We illustrate this success in the context of the private-information labor market economy.

We should perhaps reiterate here that we are following the Arrow and Debreu treatment of time and uncertainty. Our competitive contract markets operate at some initial date, and the contracts or commodities which are traded are commitments to take specified, possibly random, actions under various possible contingencies. As time evolves and observed and unobserved states of nature are realized, these commitments (contracts) are honored. Thus there are no ex post spot markets, though here these might be mutually beneficial ex post. We do not pretend to offer here a theory of the determination of market structure or legally enforceable agreements.

We begin by following Prescott and Townsend [19], defining a productionintermediation set $Y$ in such a way that $y \in Y$ and a standard market clearing condition $\sum_{i} \lambda_{i}\left(x_{i}-\xi\right)=y$ imply the resource constraint (2.3). Namely let

$$
Y=\left\{y \in L: r_{k}(y) \leq 0 \text { all } k\right\} .
$$

This production-intermediation set can be interpreted as an exchange technology where negative (positive) components correspond to a commitment to take in (distribute) resources. These commitments can be indexed by agent types; in effect, commitments can vary across agents with observable characteristics or with unobservable but declared characteristics. But the weights that agent types receive, the $r_{k}$, are fixed by the exchange technology $Y$, beyond the control of the firm-intermediary. (We shall give a more detailed interpretation of the production-intermediation set for the private-information labor market economy in what follows.) In summary, then, we use the following definition.

Definition 4.1: A competitive equilibrium is an $(I+1)$-tuple $\left(\left(x_{i}^{*}\right), y^{*}\right)$ of elements of $L$ and a price vector $p^{*} \in L$ for which (i) for each $i$ element $x_{i}^{*}$ maximizes $u_{i}(x)$ over the set $\left\{x \in \bar{X}: p^{*} \cdot x \leq p^{*} \cdot \xi\right\}$; (ii) element $y^{*}$ maximizes $p^{*} \cdot y$ over the set $Y$; and (iii) $\sum_{i} \lambda_{i} x_{i}^{*}=y^{*}+\xi$.

Existence and optimality of competitive equilibria can be established quite generally. In Prescott and Townsend [19] we established in some detail the existence of a competitive equilibrium for a private-information securities economy. Moreover, the proof given there, which relies heavily on the now standard existence arguments as in Arrow-Hahn [3], Debreu [9], McKenzie [17], and others, is applicable to other economies. We shall give, in what follows, a more direct proof of the existence of a competitive equilibrium for the private-information labor market economy, emphasizing the economic interpretation and taking advantage of the representative agent construct. (We might well have chosen the moralhazard insurance economy.) But we reiterate that ex ante diversity of the kind illustrated in the securities economy is not a problem for existence of competitive 
equilibria. As for optimality, we note that in the space of lotteries, consistent with our general environment, preferences and the consumption set are convex. Thus by Debreu [8], competitive equilibrium allocations are Pareto optimal. Again, this will be established directly in what follows for the private-information labor market economy. Again, more general arguments are contained in Prescott and Townsend [19].

To begin the discussion for the private-information labor market economy, let a generic point in the (underlying) commodity space be an output, labor pair denoted $(c, l)$ and assume as before that there are a finite number of such pairs. Now suppose that prior to the resolution of uncertainty, that is, in the planning period market, each of the finite number of firms is allowed to make commitments to the market (say, to brokers or marketeers) to supply any number of units of any such $(c, l)$ pair. More formally, let $y_{\theta}(c, l)$, if positive, denote the number of commitments to produce $c$ units of output for and to hire $l$ units of labor from households who announce they are of type $\theta$ (such announcements are public information). Each of the firm-intermediaries, then, is constrained by a production-intermediation possibilities set $Y$ defined by

$$
Y=\left\{y=\left\{y_{\theta}(c, l)\right\}: \sum_{\theta} \lambda_{\theta} \sum_{(c, l)} y_{\theta}(c, l)(a l-c) \geq 0\right\} .
$$

In effect (4.2) states that each firm-intermediary cannot plan to distribute on average more of the consumption good than it produces on average. (Note that each firm-intermediary takes the coefficients or weights in $Y$ as given, beyond its control.) The actions of the firm-intermediary are priced in competitive markets and these prices are taken as given. That is, let $p_{\theta}(c, l)$ be the per unit price of the $y_{\theta}(c, l)$ commitment in terms of some abstract unit of account. Then the firm-intermediary acts to maximize profits,

$$
\sum_{\theta} \sum_{(c, l)} y_{\theta}(c, l) p_{\theta}(c, l)
$$

subject to $y \in Y$. Recall that we are supposing firm-intermediaries are owned by households, but again, with the constant returns to scale assumption, profits will be zero and profit shares are disregarded. And again we may act as if there were only one firm-intermediary.

Households also make commitments to the market over output, labor pairs but with a different interpretation. Households are imagined to choose ex ante, in the planning period market, a contract with options, indexed by $\theta \in \Theta$. Each option is a (possibly degenerate) lottery over consumption, labor supply pairs, and the household can choose the terms of the lottery as well. But the options are such that a household of type $\theta$ ex post will choose ex post the option indexed by $\theta$ if indeed it suffers shock $\theta$. Again, see the motivating remarks in Section 2. Finally, of course, all the contracts are priced in a competitive market. More formally, let the consumption set $\bar{X}$ and endowment $\left\{\xi_{\theta}(c, l)\right\}$ be as defined in Section 2 . Then the objective of the representative household is to maximize ex ante 
expected utility

$$
\sum_{\theta} \lambda_{\theta} \sum_{(c, l)} x_{\theta}(c, l) U_{\theta}(c, l)
$$

by choice of $x \in \bar{X}$ subject to the budget constraint

$$
\sum_{\theta} \sum_{(c, l)} p_{\theta}(c, l) x_{\theta}(c, l) \leq \sum_{\theta} \sum_{(c, l)} p_{\theta}(c, l) \xi_{\theta}(c, l) .
$$

To reiterate, each household purchases a contract as a package, and does not really purchase the individual components separately. But the valuation of a contract is determined by the sum of the valuations of its individual components.

To be noted again is that the economy here is decentralized by the price system in the usual way. Thus to ensure consistency of the actions taken by households and the firm-intermediary, a market clearing condition is needed:

$$
y_{\theta}(c, l)=x_{\theta}(c, l)-\xi_{\theta}(c, l) \quad \text { all } \quad \theta \in \Theta, \quad \text { all }(c, l) \text { pairs. }
$$

Note that condition (4.6) when substituted into the production-intermediation set (4.2) yields the resource constraint.

It is now easily established that a competitive equilibrium exists and is optimal. First note from the profit maximization hypothesis and constant returns to scale condition that

$$
p_{\theta}^{*}(c, l)=\lambda_{\theta}(c-a l) \quad \text { all } \quad \theta \in \Theta, \quad \text { all }(c, l) \text { pairs, }
$$

is the only possible candidate for the equilibrium price system up to some arbitrary normalization. (We have, in effect, let the consumption good be the numeraire.) This price system on bundles $(c, l)$ has a natural interpretation. From the standpoint of the firm-intermediary, prices of bundles increase with higher output $c$ at rate $\lambda_{\theta}$ (that is, revenue increases) and prices decrease with higher labor input $l$ at rate $\lambda_{\theta} a$ (that is, cost increases). Moreover, the tradeoff under this price system between labor and output is the parameter $a$, so in effect we have marginal productivity pricing. We note of course that bundles for use by different household types can be priced differently. From the standpoint of households' budget constraints, increases in consumption $c$ raise expenditures while increases in labor supplied $l$ raise income. The price of a $(c, l)$ bundle for use under the $\theta$-option is $\lambda_{\theta}(c-a l)$ while the price of a $(c, l)$ bundle with probability $\alpha$ is $\alpha \lambda_{\theta}(c-a l)$; that is, the price system is linear in lotteries. ${ }^{5}$

Substitution of the $p_{\theta}^{*}(c, l)$ into the budget constraint for the household yields the linear program which determines Pareto optima. Thus, a solution exists and is Pareto optimal, and competitive equilibrium condition (i) is satisfied. Then setting $y_{\theta}^{*}(c, l)=x_{\theta}^{*}(c, l)-\xi_{\theta}(c, l)$, market clearing condition (iii) is satisfied. Finally, substitution of $p_{\theta}^{*}(c, l)$ into profits (4.3) makes clear that profits are nonpositive. But the budget constraint (4.5) is satisfied as an equality, so in fact profits are zero under $y_{\theta}^{*}(c, l)$. Thus condition (ii) is satisfied.

${ }^{5}$ Evidently the price system is linear in underlying commodities $c$ and $l$ for fixed $\theta$, but may vary with $\lambda_{\theta}$ and in that sense is nonlinear. 
How will the competitive equilibrium allocation be effected? As is standard in general equilibrium competitive analysis, the market assignment process and the price-determination process are unspecified. To be more specific though, we might suppose that there are a finite number of marketeers or labor-brokers which make up the planning period market. Each broker has called out the competitive equilibrium price vector and has attracted a representative pool of workers and one firm-intermediary (the brokers are really households themselves). Each household in the pool of the broker has entered into the contract $\left\{x_{\theta}^{*}(c, l)\right\}$. The firm has committed itself to input-output vector $\left\{y_{\theta}^{*}(c, l)\right\}$. Next, in the consumption period, each household truthfully announces its shock $\theta$. If $\left\{x_{\theta}^{*}(c, l)\right\}$ is not degenerate in labor supply, then the broker uses some random sorting device to select workers from its pool in the proper proportions. These are directed to the firm consistent with planning period demand. The consumption good is then produced and distributed by the broker to all workers in the pool in accordance with $\left\{x_{\theta}^{*}(c, l)\right\}$, as if under a wage-benefit or insurance package. We are assuming, of course, that planning period contracts are honored (or costlessly enforced by a legal system); that is, there can be no collusion in labor supply among households (say to eliminate risk) and no ex post spot markets.

Now suppose an outside observer were to see the realized outcome of a competitive equilibrium allocation. We have just established that such an allocation is optimal, so we know its properties from Section 3. But these properties might lead the outside observer to conclude that the realized outcome is inconsistent with the competitive market paradigm: if labor and output are fully observed, then the constant marginal product of labor might be inferred. That then might naturally be taken as the wage rate. If households were asked to indicate whether they would like to work more at that wage rate, some would so indicate. Thus there would be measured unemployment (nonmarket clearing). Moreover, the outside observer might see disparities in employment among households who indicate they are otherwise identical, as if employment were capricious or random. Conceivably, some households might be completely unemployed, while others work up to maximum capacity. But, again, such outcomes are not inconsistent with the competitive market paradigm if the objects which are traded are carefully defined. As we have indicated, such outcomes can be optimal from the ex ante point of view of the representative household, and can be achieved in competitive markets for labor contracts with appropriately specified options. We have, in effect, taken a step toward a synthesis implicit labor contract theory with standard competitive analysis.

\section{STANDARD COMPETITIVE EQUILIBRIA IN ECONOMIES WITH ADVERSE SELECTION: THE DIFFICULTIES OF PRICE DECENTRALIZATION}

Unlike the moral-hazard insurance and private-information labor market economies, the resource constraints in the adverse-selection insurance and signaling economies are of the form

$$
\sum_{i} \lambda_{i} r_{i k} \cdot\left(x_{i}-\xi\right) \leq 0
$$

$$
(k=1, \ldots, K)
$$


rather than of the less general form

$$
\sum_{i} \lambda_{i} r_{k} \cdot\left(x_{i}-\xi\right) \leq 0
$$

$$
(k=1, \ldots, K)
$$

This turns out to be a very important difference, for production and consumption activities cannot be separated as before. ${ }^{6}$ To see this, let

$$
Y=\left\{y \in L: \sum_{i} \lambda_{i} r_{i k} \cdot y \leq 0, k=1, \ldots, K\right\}
$$

be the candidate for the production-intermediation possibilities set. If $r_{i k}=r_{k}$ for all $i$, then the production-intermediation set (5.3) is the productionintermediation set (4.1) used previously. In that case market clearing, i.e.,

$$
y=\sum_{i} \lambda_{i}\left(x_{i}-\xi\right)
$$

and $y$ belonging to $Y$ imply the resource constraint. But, in general, (5.1), (5.3) and (5.4) are inconsistent.

As it turns out, three natural ways to circumvent this adverse-selection problem all fail to decentralize the economy in the usual way. In this section we summarize what we have learned in these attempts. For a more detailed discussion, the reader is referred to the working paper, Prescott and Townsend [20].

One attack on the inconsistency (adverse-selection) problem is to ignore it, with the hope that it fails to occur in equilibrium, in a standard competitive equilibrium, that is, in which decisions are completely decentralized by a price system. Thus, one might make use of the production-intermediation set (5.3) in the obvious commodity spaces. For the adverse-selection insurance economy, the obvious commodity space is the space of loss, no-loss consumption bundles. But that with (5.3) implies the same budget line for all household types, the (average) market-odds line of Rothschild-Stiglitz, and that with diversity across household-types implies the nonexistence of a standard competitive equilibrium. For the Spence signaling economy, the obvious commodity space is the space of commodity triplets specifying consumption, signal, and labor supply. But that with (5.3) implies a zero price for the signal, with labor priced in terms of the consumption good at the average productivity. Thus, a standard competitive exists for the Spence signaling economy, but it involves no signaling, the phenomenon which the model was intended to explain.

Motivated by these considerations one might hope to allow different agent types to choose different commodity bundles in equilibrium. Hence, we expanded the commodity space in an obvious way. Namely, for the Spence signaling economy we supposed different signals could be treated as different

\footnotetext{
${ }^{6}$ There would be separation in the signaling economy if markets opened prior to individuals knowing their types. Thus, signaling opportunities per se are not the cause of failure. Similarly, this failure would have arisen in the environment of Prescott-Townsend [19] if there were some statistical dependence in the $\theta$-shocks and the initial specification of $i$ types. And there would be a problem in the moral-hazard insurance economy if it were combined with the adverse-selection insurance economy.
} 
commodities, each with a potentially distinct price in terms of the consumption good. We also introduced a production-intermediation set, with coefficients in the technology which firms treat parametrically, but which in fact are functions of the households' equilibrium consumption choices. We then defined a standard competitive equilibrium with production externalities and established that all the Spence signaling equilibria are examples of equilibria of that kind, including the no-signaling equilibria, the minimal-signaling equilibria, and the over-signaling equilibria. Of course, many of these are Pareto nonoptimal; indeed, many can be Pareto ranked. We also established that an analogous procedure could be applied to the adverse-selection insurance economy to support a bewildering variety of standard competitive equilibria (with production externalities). Thus equilibria of this kind fail to provide much predictive content and have undesirable normative properties as well.

We also expanded the commodity space in a different way, following the spirit of Arrow [1], with the intent of removing externalities. More specifically, we indexed the original commodity point in the general structure by $i$, thereby increasing the dimensionality by factor $I$. Again, the new commodity point has the interpretation of a contract with options which are effected by the individual households. In this expanded space there is a specification of the productionintermediation set (without externalities) which is always consistent with market clearing and the resource constraints. Thus one can consider an extension of the standard support theorem for Pareto optimal allocations. In fact, we established that there is a price system such that every Pareto optimum can be supported in a kind of competitive equilibrium. But the competitive equilibrium is restricted in that the assignment in the optimum to other agent types must be taken into account in each household's maximization problem. In effect, then, there are externalities in consumption. Moreover, the same support theorem can be used to establish that unrestricted competitive equilibria in the indexed commodity space generally do not exist for the signaling and adverse-selection insurance economies.

We conclude that there do seem to be fundamental problems for the operation of competitive markets for economies or situations which suffer from adverse selection. We have not discovered a standard competitive equilibrium construct which would predict well in such situations.

\section{University of Minnesota \\ and \\ Carnegie-Mellon University}

Manuscript received April, 1980; final revision received December, 1982.

\section{REFERENCES}

[1] Arrow, Kenneth J.: "The Organization of Economic Activity: Issues Pertinent to the Choice of Market vs. Nonmarket Allocation," in The Evaluation and Analysis of Public Expenditures: The PBB System. Joint Economic Committee Compendium, 91 Congress 1st Session, Vol. 1, 1969. 
[2] —-: "The Role of Securities in the Optimal Allocation of Risk Bearing," Review of Economic Studies, 31(1964), 91-96.

[3] Arrow, Kenneth J., and Frank Hahn: General Competitive Analysis. San Francisco: Holden Day, 1971.

[4] Azariadas, Costas: "Implicit Contracts and Underemployment Equilibria," Journal of Political Economy, 83(1975), 1183-1202.

[5] Bailey, Martin N.: "On the Theory of Layoffs and Unemployment," Econometrica, 45(1977), $1043-1064$.

[6] Bewley, Truman, and Roy Radner: "Stationary Monetary Equilibrium with a Continuum of Independently Fluctuating Consumers," Northwestern University Working Paper, October, 1980.

[7] Chari, V.: "Involuntary Unemployment and Implicit Contracts," Quarterly Journal of Economics, 98(1983), 107-122.

[8] Debreu, Gerard: "Valuation Equilibrium and Pareto Optimum," Proceedings of the National Academy of Science, 40(1954), 588-592.

[9] —_ "New Concepts and Techniques for Equilibrium Analysis," International Economic Review, 3(1962), 257-273.

[10] - The Theory of Value. New York: Wiley, 1959.

[11] Gordon, Donald: “A Neo-Classical Theory of Keynesian Unemployment," Economic Inquiry, (1974), 431-459.

[12] Green, Jerry, and Charles Kahn: "Wage Employment Contracts," Quarterly Journal of Economics, 98(1983), 173-188.

[13] Grossman, Stanford, and Oliver Hart: "Implicit Contracts under Asymmetric Information," Quarterly Journal of Economics, 98(1983), 123-156.

[14] Harris, Milton, and Robert M. TownSend: "Allocation Mechanisms for Asymmetrically Informed Agents," Carnegie-Mellon Working Paper 55-76-77, March, 1977.

[15] -: "Resource Allocation Under Asymmetric Information," Econometrica, 49(1981), 33-64.

[16] Hurwicz, L.: "On Informationally Decentralized Systems," in Decision and Organization, edited by R. Radner and B. McGuire. Amsterdam: North-Holland, 1971.

[17] McKenzie, L.: "On the Existence of General Equilibrium for Competitive Markets," Econometrica, 27(1959), 54-71.

[18] Myerson, Roger: "Incentive Compatibility and the Bargaining Problem," Econometrica, 47(1979), 61-74.

[19] Prescott, Edward C., and Robert M. Townsend: "General Competitive Analysis in an Economy with Private Information," June, 1979, revised August, 1982.

[20] - "Optima and Competitive Equilibria with Adverse Selection and Moral Hazard," June, 1981.

[21] Rothschild, Michael, and Joseph Stiglitz: "Equilibrium in Competitive Insurance Markets: An Essay on the Economics of Imperfect Information," Quarterly Journal of Economics, 90(1976), 629-649.

[22] SPEnCE, A. Michael: Market Signaling: Information Transfer in Hiring and Related Screening Processes. Cambridge: Harvard University Press, 1974.

[23] Wilson, Charles: "A Model of Insurance Markets with Incomplete Information," Journal of Economic Theory, 16(1977), 176-207. 